\title{
1 From Conventional to Miniaturized Analytical Systems
}

\author{
Francisco Pena-Pereira \\ Analytical and Food Chemistry Department; Faculty of Chemistry; \\ University of Vigo, Campus As Lagoas-Marcosende s/n, 36310 Vigo, Spain \\ e-mail address: fjpena@uvigo.es
}

\subsection{Introduction}

Nowadays, the term miniaturization is applied to a wide spectrum of knowledge areas, including, among others, engineering, physics, medicine, materials science, computer science and chemistry. A search on the ISI Web of Knowledge provided approximately 42000 results by entering the term miniaturization, from which around 5200 results are devoted to chemistry. The number of publications concerning the miniaturization of chemical systems has experienced an important increase in the last two decades, as has the number of citations received by these publications, as shown in Figure 1.1. In accordance with the ISI Web of Knowledge, they currently receive around 12000 citations per year. Nevertheless, this is only the tip of the iceberg since the number of publications devoted to the development and application of miniaturized analytical systems (but not referring to miniaturization in the title or abstract sections) are not included.

In the broadest sense of the word, miniaturization can be defined as the production of novel systems that are substantially reduced in size in comparison with conventional systems. In analytical chemistry, the term miniaturization does not refer solely to the scaling-down of analytical instrumentation, apparatus and devices since it is also applicable when the components (including chemicals and solvents) needed to perform analytical operations are employed on a greatly reduced scale. In fact, size reduction is not the main driving force when shrinking analytical systems, as can be deduced from section 1.2. It is worth noting that the term miniaturization has been mainly employed in the analytical chemistry literature to refer to the micro-total analysis systems ( $\mu$-TAS) and lab-on-a-chip (LOC) devices. Even though they represent the highest degree of downsizing, the concept of miniaturization should be observed from a broader, non-exclusive perspective since this concept includes the advances achieved in every single step of the analytical process.

A recent trend in analytical chemistry is a progression towards the miniaturization of analytical systems. Different steps of the analytical process, including sample preparation, analytical separation and detection have been subjected to miniaturization, automation and portability. In addition, the integration of different analytical steps has allowed the development of fully miniaturized systems. The miniaturiza- 

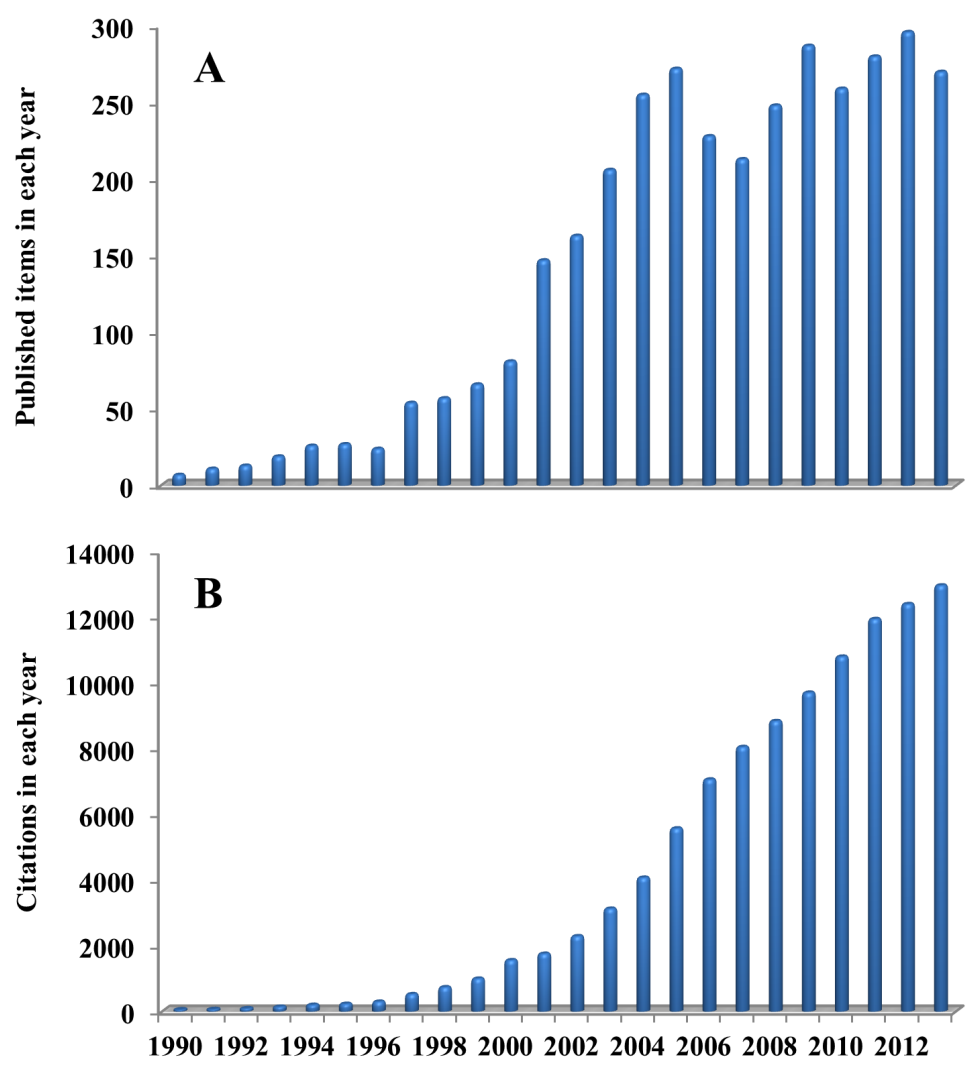

Figure 1.1 (A) Evolution of the publications devoted to miniaturization in chemistry; (B) Citations received by the publications devoted to miniaturization in chemistry (source: ISI Web of Knowledge (Web of Science) - Thomson Reuters).

tion of analytical systems has been mainly addressed as a result of the necessity to overcome problems and meet the demands from novel research areas. As a result, challenging requirements have been established for analytical microsystems, namely the analysis of highly reduced sample volumes with the highest possible sensitivity, selectivity and precision in reduced analysis times, with reduced amounts of reagents and/or organic solvents and, whenever possible, in the field.

In this chapter, an overview of the main advances towards the miniaturization of conventional systems that are commonly employed in analytical chemistry is provided. Considerations on the miniaturization of the three most developed steps of the analytical process, namely sample preparation, analytical separation and detection are provided and representative applications are briefly described. 


\subsection{Miniaturizing Steps in the Analytical Process}

As early as 1959, Feynman introduced a field focused on the problem of manipulating and controlling things on a small scale in his inspiring talk entitled 'There is plenty of room at the bottom' (Feynman, 1992). Feynman predicted that the improvement of several scientific subjects by means of miniaturization would allow scientists to better understand fundamental problems and to overcome the limitations of fullsized devices. Miniaturization of analytical instrumentation was identified as a significant trend in the field by the journal Analytical Chemistry in 1970 (Senzel, 1970). Improvement and size reduction of analytical instrumentation and devices has been a challenge for several decades. In the last two decades the analytical scientific community has experienced a renewed growth of interest in miniaturized systems concerning the different analytical process steps, mainly influenced by the introduction of microextraction techniques, miniaturized separation techniques and detection systems, as well as the development of $\mu$-TAS and the novel generations of flow injection techniques. The miniaturization of analytical systems is generally linked to other challenges in analytical chemistry, such as portability, automation and greening of analytical procedures. In addition, economy, rapidity, improved analytical performance and the size decrease of analytical systems are among the drivers for miniaturization (Figure 1.2).

The analytical process involves all the steps needed to obtain analytical information from a sample, namely sample collection and preservation, sample preparation, separation, detection, data processing and final decision (Figure 1.3). Today, almost every step of the analytical process has been subjected to miniaturization. However, the different steps of the analytical process have not been miniaturized to the same extent. For instance, sample collection and preservation is the step of the analytical process less subjected to the benefits of miniaturization, even though some autonomous and remote sensing analytical microsystems have been reported. Conversely, data acquisition and processing have achieved an excellent degree of miniaturization. Furthermore, it is generally accepted that the downscaling of sample preparation approaches has been developed after certain efforts to miniaturize both separation and detection systems.

It is worth mentioning that full miniaturization of analytical systems has also been addressed in the literature. Thus, two different systems, namely $\mu$-TAS and labon-a-valve (LOV), enable the miniaturization of the different steps needed to perform a chemical analysis in a single system. The concept of $\mu$-TAS was firstly introduced two decades ago by Manz et al. with the aim of enhancing the analytical performance of total analysis systems (TAS) rather than a simple reduction of their size (Manz et al., 1990a). In this pioneering work, the authors defined $\mu$-TAS as "TAS systems that perform all sample handling steps extremely close to the phase of measurement". The introduction of surface techniques amenable to achieve mechanical microstructures has been critical in the development of $\mu$-TAS systems. Nowadays, $\mu$-TAS systems are 


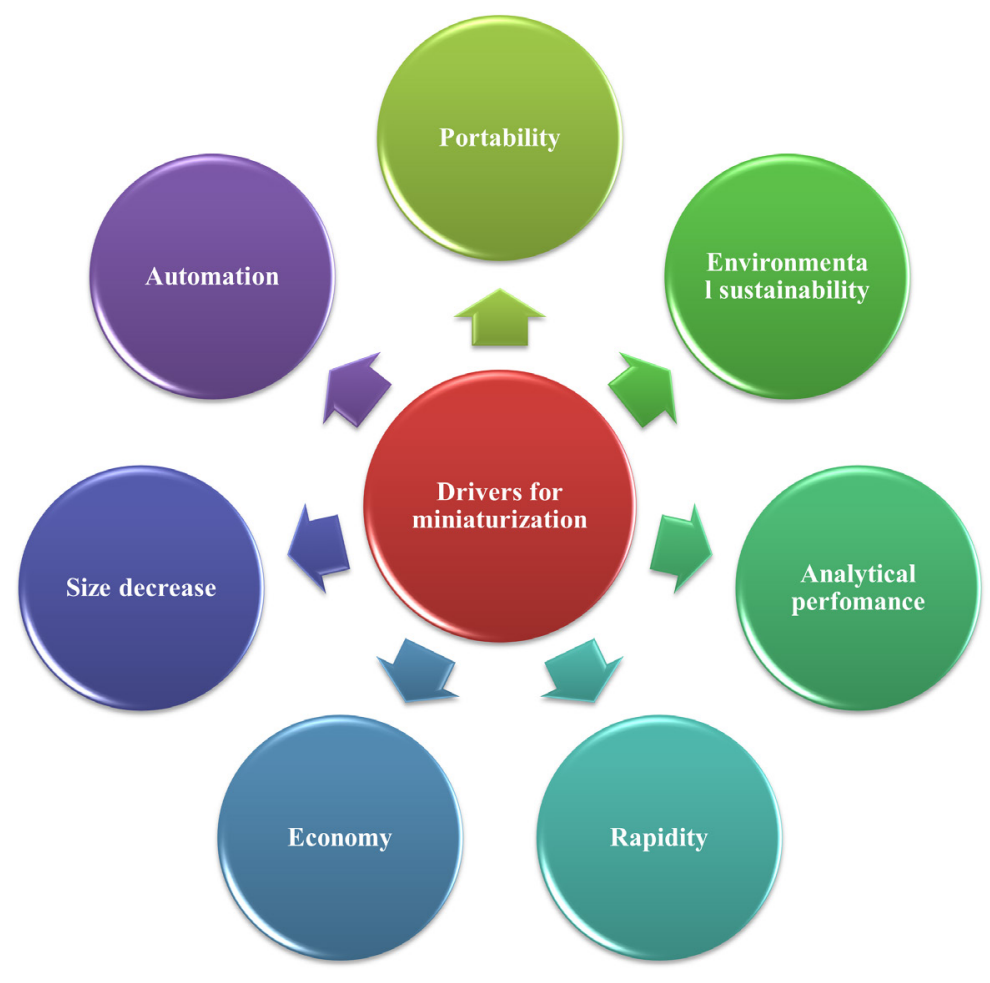

Figure 1.2 Drivers towards the miniaturization of analytical systems.

not a fashionable craze, but a powerful and exciting interdisciplinary research field (Marx et al., 1991). A variety of excellent books (Herold \& Rasooly, 2009; Ríos et al., 2009; Lin, 2011) and reviews (Mark et al., 2010; Livak-Dahl et al., 2011; Rios et al., 2012; Nge et al., 2013) on microfluidics are available in the literature for interested readers. More recently, Ruzicka introduced the concept of LOV as a versatile methodology for downscaling reagent-based (bio)chemical assays to micro- and submicroliter level (Ruzicka, 2000). The micro-sequential injection ( $\mu$ SI)-LOV system, also reported as the third generation of flow injection analysis systems, enables one to carry out the unitary steps needed to perform an analysis on the basis of the use of a central sample processing unit. A number of review articles covering the evolution of flow injection techniques have been published (Hansen \& Wang, 2005; Idris, 2010; Yu et al., 2011).

Size reduction is not the only reason towards miniaturization. In fact, the miniaturization of the different steps of the analytical process involves several additional benefits, as can be seen in Figure 1.4. The main benefits that can be obtained by downsizing the different steps of the analytical process are shown below:

1. Reduction of sample amount: The sample volume required to carry out an appropriate analysis can be highly reduced by scaling down the sample preparation, 


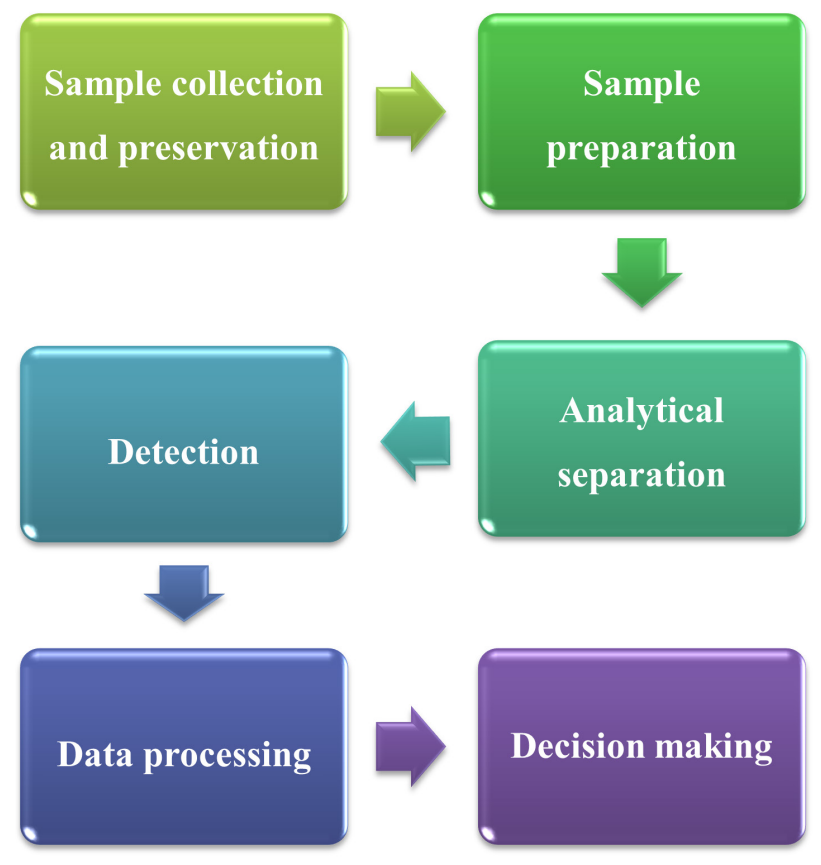

Figure 1.3 Steps of the analytical process.

separation and detection techniques. This is especially advantageous when dealing with scarce and/or precious samples.

2. Decreased consumption of chemicals and solvents: A drastic decrease in the amount of analytical reagents and organic solvents that are needed can result from the miniaturization of any analytical process step. This is especially important in the case of analytical methods involving expensive and precious reagents such as enzymes and immunochemicals, as well as in the case of analytical methodologies involving toxic reagents and/or organic solvents. Certain sample preparation strategies even allow the total removal of organic solvents and reagents, thus contributing to the environmental sustainability of analytical laboratories. The miniaturization of separation techniques enables a significant reduction of mobile phase or electrolyte, as well as the amount of stationary phase materials. As for detection techniques, reagent and gas consumption savings can be significant. In this sense, the reduction of neutral gas consumption is certainly remarkable in the case of miniaturized plasma sources.

3. Reduction of associated wastes: As a result of the above mentioned advantages, the wastes generated along the whole analytical process can be highly reduced, thus resulting in more sustainable methodologies. Recycling and recovery of chemicals and organic solvents present in wastes, as well as the on-line generation of clean wastes are important tasks aiming to be adopted in analytical laboratories (Garrigues et al., 2010). 

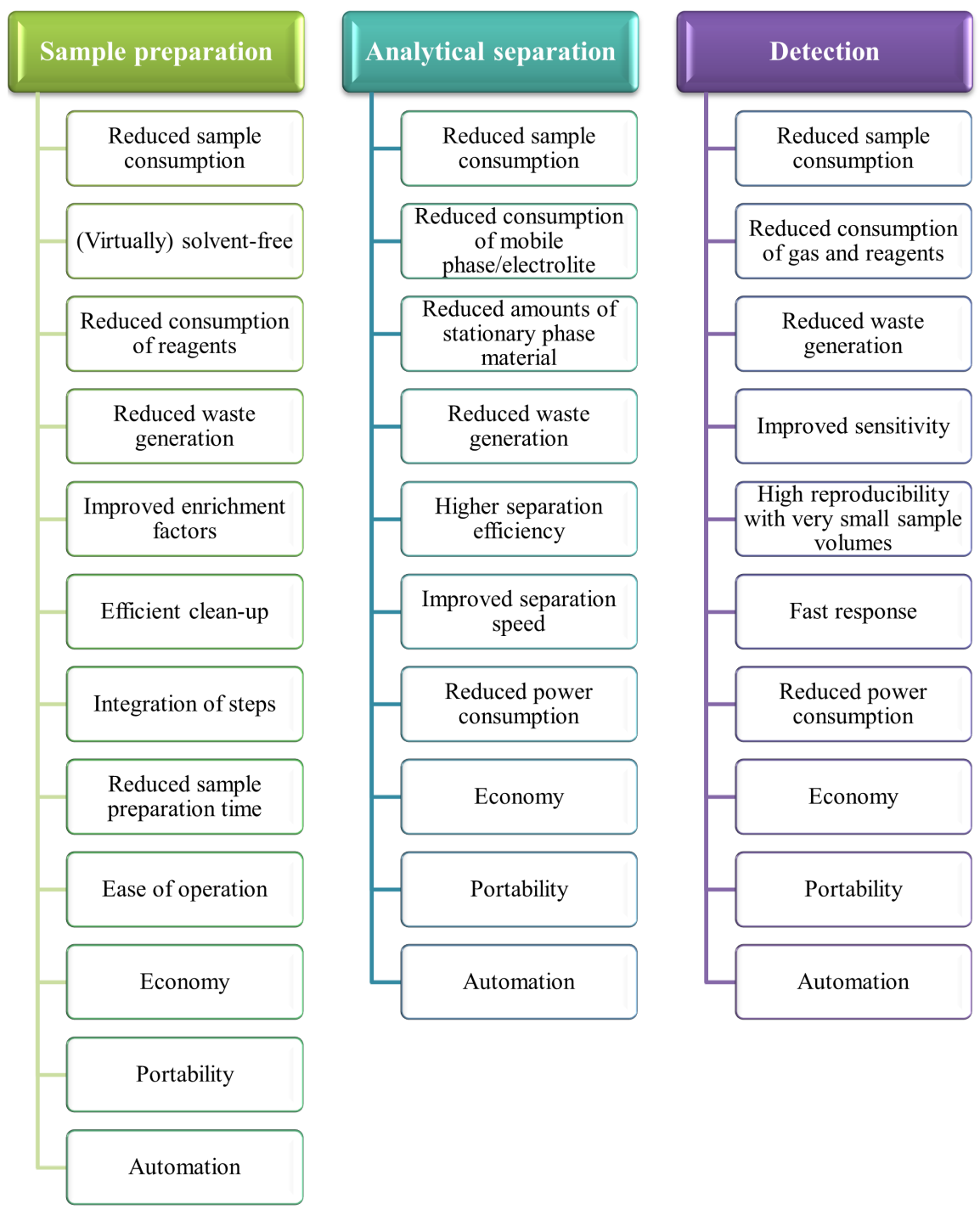

Figure 1.4 Potential benefits derived from the miniaturization of the different steps of the analytical process.

4. Improved sensitivity: Sensitivity of analytical methods can be increased by making use of an appropriate miniaturized sample preparation technique and, in certain cases, by miniaturizing detection systems. In sample preparation, high enrichment factors (EFs) can potentially be obtained as a result of the increased sample volume-to-extractant phase ratio, which can result in lower limits of detection. The improved design of recently developed analytical instrumentation can also yield increased sensitivity by using reduced sample volumes, although 
in several cases the sensitivity can be significantly deteriorated when the instrumentation is miniaturized. Advances in detection systems can minimize the loss of sensitivity.

5. Rapidity: Time is a vital variable in analytical laboratories. The sample throughput is therefore an important factor in method development. The development of miniaturized sample preparation, separation and detection systems can significantly decrease the time needed to perform a single analysis. Miniaturization allows the improvement of the two most time-consuming steps, namely sample pre-treatment and analytical separation. Apart from the obvious benefit of having access to analytical data in an expeditious way, the reduction of the analysis time can provide indirect benefits, for example reduced consumption of reagents and solvents, lower energy requirements and smaller amounts of waste.

6. Portability: The development of field-portable instrumentation has been and is still a challenge in analytical chemistry. Low weight and overall dimensions, being resistant to changeable environmental conditions and efficient battery power are the requirements for portable analytical systems. The miniaturization of part or the whole of the analytical process steps contributes significantly to the portability of analytical systems to the sampling site. Furthermore, portable analytical systems deliver prompt and valuable information and reduce the risk of sample decomposition and contamination during sample storage and transportation.

7. Power consumption: The reduction of analytical systems generally involves a reduction of the power requirements. As a consequence, miniaturized instrumentation can be battery-operated, then contributing to its portability.

As can be noticed, several of the above mentioned advantages are related to each other. It is important to note, however, that the introduction of miniaturized alternatives to conventionally performed steps of the analytical process can give rise to novel challenges that need to be addressed. Therefore, the miniaturization of certain analytical processes may be not just useless but counterproductive. Examples of this fact include the development of microfluidic systems when large volumes of samples are available and/or conventional methods involve the use of small amounts of non-toxic and non-expensive reagents and solvents (Luque de Castro \& Priego Capote, 2008). Besides, the use of highly reduced sample amounts can seriously affect the necessary representativeness of samples subjected to analysis. On the other hand, the fabrication of miniaturized detectors can also yield reduced resolution when compared with the corresponding full-sized counterpart (Capitan-Vallvey \& Palma, 2011).

The reduced sample volume used with miniaturized analytical separation techniques can also give rise to reduced sensitivity, since the miniaturization of the chromatographic column involves the reduction of the detector volume. For instance, the use of miniaturized liquid chromatography (LC) for the analysis of easily available samples can give rise to problems of sensitivity that could be easily circumvented with 


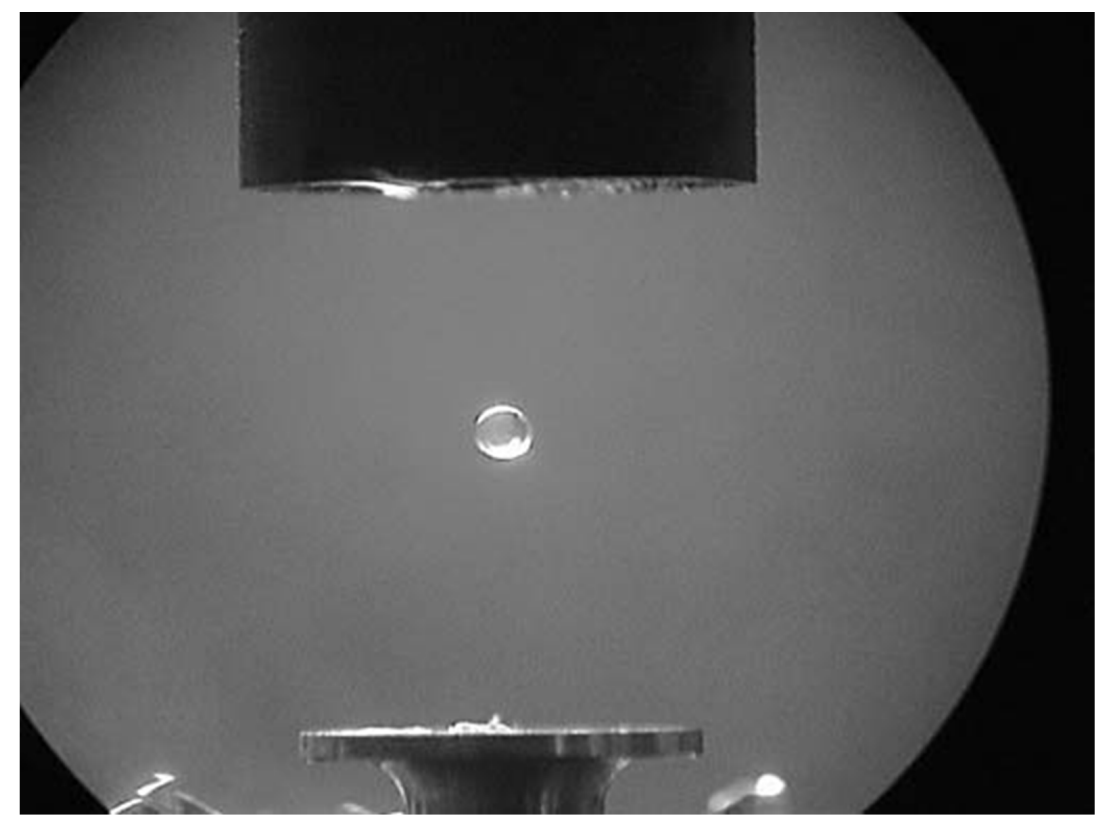

Figure 1.5 A 500-nL water drop levitated in a node in a standing wave created between an ultrasonic transducer (bottom) and a solid reflector (top). Reprinted from Santesson \& Nilsson (2004) with permission from Springer.

conventional LC (Desmet \& Eeltink, 2013). In addition, the development of novel analytical methods involving miniaturized sample preparation approaches introduces novel concerns derived from the limitations of the corresponding sample pre-treatment techniques. This can be the case where organic solvents with higher toxicity than in conventional analytical methods are used or when the introduction of numerous steps gives rise to tedious procedures and potential sources of contamination. On the other hand, the problem of adsorption to solid walls and interfaces when dealing with sample volumes in the picolitre to nanolitre range has been reported in the literature. The applicability of acoustic or ultrasonic levitation (Figure 1.5) has been proposed to avoid this problem, which can be of special concern when miniaturizing analytical and bioanalytical processes (Santesson \& Nilsson, 2004; Priego-Capote \& de Castro, 2006).

This technology, also known as lab-on-a-drop, is compatible with a variety of remote detection systems, and allows one to perform several analytical applications, including liquid-liquid and gas-liquid extraction, chemical and biochemical derivatization, solvent exchange, titration, crystallization, affinity two-phase separation and concentration by evaporation (Priego-Capote \& de Castro, 2006). Even though the number of publications concerning levitation in chemical analysis is still relatively low in analytical chemistry, it is expected that this containerless sample handling technology will have an impact on the development of novel miniaturized systems. 


\subsubsection{A Need for Scaling Down Conventional Sample Preparation Techniques}

Sample preparation is one of the most important steps of the analytical process, especially when dealing with the determination of trace (or ultratrace) analytes in complex samples. It is generally accepted that sample pre-treatment, together with sample collection and preservation, is the most time consuming and error-prone step of the analytical process. Sample preparation is employed in analytical chemistry to preconcentrate target analytes from samples where they are present at lower concentrations than the limit of detection of the corresponding analytical technique, to achieve a clean-up of the sample prior to instrumental analysis and/or to obtain an extract compatible with the analytical technique to be used.

A plethora of sample preparation techniques can be employed in analytical laboratories, including solid-phase extraction (SPE), conventional solvent extraction, Soxhlet extraction, pressurized solvent extraction, supercritical fluid extraction, and microwave- and ultrasound-assisted extraction. A variety of extractant phases, for example polymeric sorbents and adsorbents, organic solvents, ionic liquids, water or carbon dioxide can be used depending on the sample preparation technique. Furthermore, extraction processes can be enhanced by means of efficient selection of the experimental conditions. SPE and solvent extraction are, by far, the most commonly used sample preparation techniques in analytical laboratories. In fact, many official and standardized analytical methodologies involve their application for the extraction, preconcentration and sample clean-up prior to determination of target analytes. SPE and solvent extraction involve the partitioning of target analytes between the sample solution and a solid (adsorbent) phase or immiscible organic solvent, respectively. Both SPE and solvent extraction are exhaustive extraction techniques, so quantitative transfer of target analytes from the sample to the extractant phase is achieved under optimal conditions. However, the consumption of large amounts of organic solvents and subsequent generation of waste, the relatively low EFs that are achievable and the tediousness and significant time consumption are among the inherent drawbacks associated with these classical sample preparation approaches. These limitations led to the introduction of modern sample preparation techniques that share the common benefits of miniaturization and (virtually) solvent-free operation.

As early as 1990, Arthur and Pawliszyn presented the first miniaturized sample preparation technique, introduced under the denomination of solid-phase microextraction (SPME) (Arthur \& Pawliszyn, 1990). SPME is based on the partitioning of target analytes between the sample solution (or the headspace above it) and a polymeric extractant phase coated on a fused silica fiber. SPME is a non-exhaustive solvent-free sample preparation technique that allows the extraction and preconcentration of a vast number of compounds. The wide acceptance of SPME by the scientific community is reflected by the large number of publications involving this miniaturized sample preparation technique. Since its inception, SPME has been extensively employed in a variety of research fields. Related miniaturized sample preparation 
techniques have been introduced in recent years as a result of the development of novel designs, as well as the implementation of novel materials. Thus, stir bar sorptive extraction (SBSE) was developed by coating a magnetic stir bar with an appropriate sorbent coating (Baltussen et al., 1999). Importantly, the surface area and sorbent coating volume are greatly improved in SBSE when compared with SPME, thus resulting in the achievement of higher extraction efficiencies (EEs) and, when thermal desorption is performed, lower limits of detection. A variety of related sample preparation techniques have also been reported in the literature, including thin film microextraction, solid-phase dynamic extraction and microextraction in a packed syringe. Relevant theoretical and experimental aspects of SPME and related sample preparation approaches are discussed in depth in chapters 2 and 3. These sample pretreatment techniques can cover most of the current requirements in terms of extractability and selectivity, with solid phase coatings showing good thermal, chemical and mechanical stabilities.

Liquid-phase microextraction (LPME) techniques have been developed recently with the aim of improving conventional solvent extraction. Specifically, the objectives required to miniaturize the solvent extraction technique were, mainly, to reduce the relatively large organic solvent volume conventionally needed to perform a single extraction process, to obtain high EFs, and, in general, to accelerate and simplify the process, thus allowing higher sample throughput. Liu and Dasgupta (1995) and Jeannot and Cantwell (1996) reported the first works concerning the miniaturization of conventional solvent extraction. The employment of a microliter-volume single drop of extractant phase exposed at the end of a capillary or, more commonly, at the tip of a microsyringe, allowed the enrichment of target analytes from both liquid and gaseous samples. This miniaturized solvent extracton technique, named as singledrop microextraction (SDME), allows the achievement of high EFs in spite of being a non-exhaustive technique. Further advances to improve the stability of the extractant phase during the extraction process involved the use of hollow fibers (PedersenBjergaard \& Rasmussen, 1999). Even though the use of these membranes allow the employment of experimental conditions that favorably affected the mass transfer of the analyte from the sample solution to the extractant phase (mainly, high stirring rates and extended extraction times), hollow fiber-based LPME approaches have not achieved the levels of popularity of related miniaturized sample preparation techniques, probably due to the insufficient EEs as well as its increased level of manipulation when compared with SDME. The development of dispersive liquid-liquid microextraction (DLLME) by Rezaee et al. in 2006 expanded the applicability of LPME as a result of its simplicity and the quantitative extraction recoveries achieved (Rezaee et al., 2006). DLLME is based on the use of a disperser solvent in combination with a water-immiscible extractant phase. The disperser acts as a bridge between the sample solution and the solvent. It allows the formation of tiny microdrops of the extractant phase that can disperse through the sample, then improving the mass transfer of the analyte towards the acceptor solution. Separation of the involved phases can 
be achieved easily by centrifugation on the basis of their different densities. Several related LPME approaches have also been reported, such as directly suspended droplet microextraction, cold induced aggregation microextraction and solidified floating organic drop microextraction. An in-depth discussion on the different LPME techniques, as well as on the different extractant phases that can be employed in LPME, is provided in chapters 4 and 5.

It is worth noting that certain sample preparation modes allow the improvement of selectivity by exploiting the physicochemical properties of target analytes making use of appropriate derivatization reactions, by exploiting kinetic discrimination, or by means of novel materials and/or separation membranes. Another advantage of miniaturized sample preparation approaches lies in the possibility of performing the extraction and derivatization of target analytes simultaneously. The integration of these unit operations reduces the number of steps that are necessary to carry out the analysis, and increases sample throughput. It should be kept in mind, however, that the possibility of integrating the extraction process and the derivatization reaction in a single step is obviously dependent on the compatibility of both processes. The advances on sample preparation towards their miniaturization are discussed in greater detail in subsequent chapters.

\subsubsection{Miniaturization of Analytical Separation}

Analytical separation techniques are employed in analytical chemistry for the separation of target analytes prior to their detection. A variety of separation techniques have been developed with the aim of separating and identifying a large number of compounds, with LC, gas chromatography (GC) and capillary electrophoresis (CE) being the most commonly employed analytical separation techniques.

In general, the miniaturization of the analytical separation techniques attempts to increase the separation efficiency and speed of the separation, decrease the cost and enhance portability, as well as reduce the amount of sample, solvent and reagents consumed and the wastes generated during the separation process. The first step towards the miniaturization of analytical separation techniques has been the result of the natural necessity of saving space in the laboratory. In fact, the first available analytical separation techniques were largely oversized, while much smaller analytical separation systems are currently available (Bartle \& Myers, 2002). Further miniaturization has been the result of the proper search for solutions needed for challenging research activities, together with the developments on related areas, such as instrumental engineering and material sciences. The development of advanced fabrication technologies, such as micro-electromechanical systems (MEMS), has also been critical to miniaturizing analytical separation techniques. 


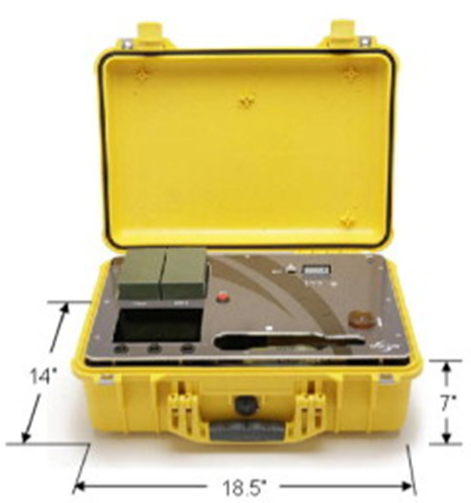

(A)

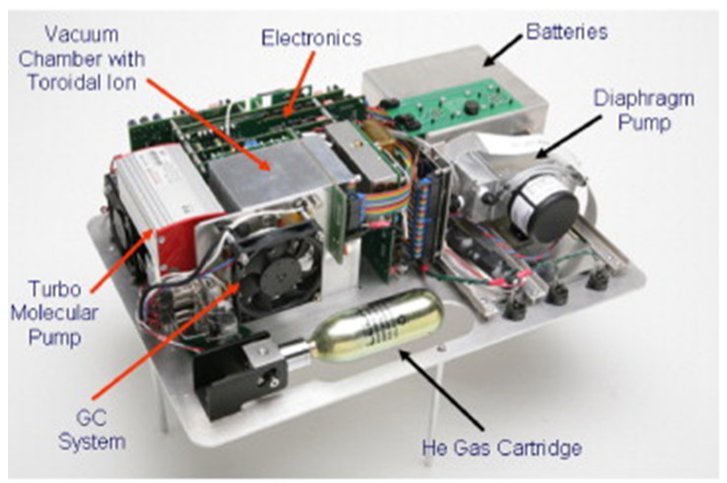

(B)

Figure 1.6 Photographs of the Guardion-7 GC-TMS showing (a) dimensions and (b) internal components. Reprinted from Contreras et al. (2008) with permission from Springer.

\subsubsection{Gas Chromatography}

GC is a standard analytical separation technique that allows the separation of complex mixtures of volatile and semi-volatile compounds. First introduced by James and Martin in the early 1950s (James \& Martin, 1952), the introduction of capillary columns in GC was the first step toward the miniaturization of analytical separation techniques (Golay, 1958). GC systems have decreased in size to a great extent in recent years. For instance, a personal field portable GC system that combines a low temperature thermal mass injector, a low temperature thermal mass capillary GC and a miniature toroidal ion trap mass analyzer (TMS) has been reported. The proposed GC-TMS system (Figure 1.6) includes carrier gas supply and battery power source, has a relatively low weight ( $<13 \mathrm{~kg}$, including batteries) and is totally self-contained within dimensions of $470 \times 360 \times 180 \mathrm{~mm}$ (Contreras et al., 2008).

The production of miniature gas chromatographs is of high interest not only in analytical chemistry, but also in other scientific and technological fields. The devel- 


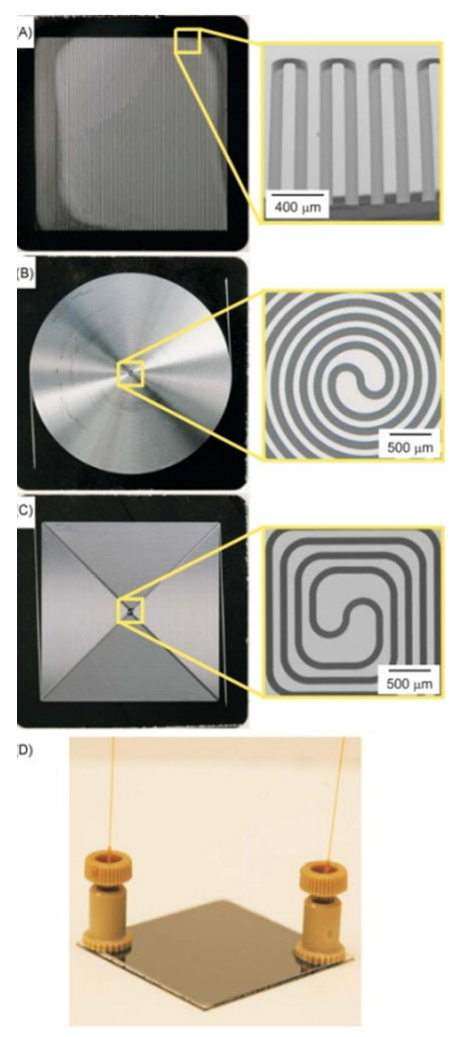

Figure 1.7 Photograph showing three different silicon-Pyrex ${ }^{\circledR}$ microcolumn configurations tested: circular-spiral with $75 \mu \mathrm{m}$ distance between channels (A), square-spiral (B), and serpentine with $100 \mu \mathrm{m}$ separation distance between channels (C). Each microcolumn shown is $3 \mathrm{~m}$ long and $100 \mu \mathrm{m}$ $\times 100 \mu \mathrm{m}$ in cross-section. Fused silica capillaries $(200 \mu \mathrm{m}$ outer diameter, $100 \mu \mathrm{m}$ internal diameter) were attached to the microcolumn chip via Nanoports ${ }^{\circledR}$ (D). Reprinted from Radadia et al. (2010) with permission from Elsevier.

opment of the first chip-based GC system was reported as early as 1979, when Terry et al. fabricated a miniaturized GC on a $5 \mathrm{~cm}$-diameter silicon wafer by using photolithography and chemical etching techniques (Terry et al., 1979). The system presented the components of a conventional GC, namely a carrier gas supply, sample injection system, separation column $(1.5 \mathrm{~m}$ ) and output detector (thermal conductivity detector), but in highly reduced dimensions. The preparation of the stationary phase of the proposed micro gas chromatograph ( $\mu$-GC) resulted, however, in relatively poor separation performance when compared with conventional GC systems. To improve this pitfall, the geometry of $\mu$-GC columns is currently the focus of study (Mittermuller \& Volmer, 2012). A recent article has revealed that a microfabricated serpentine geometry provides an improved separation performance when compared with circular- 
spiral and square spiral channel configurations in GC microcolumns (Figure 1.7) (Radadia et al., 2010).

$\mu$-GC systems are known for their low size and weight. For instance, Figure 1.8 shows a complete $\mu$-GC system with highly reduced dimensions (300 x 170 x $80 \mathrm{~mm}$ ) and a weight less than $3 \mathrm{~kg}$, consisting of a multi-stage preconcentrator/injector, a capillary column and a photoionization detector (Jian et al., 2013).

Complete $\mu$-GC systems have been developed on account of the advances achieved in downsizing their individual components, such as micropumps, micropreconcentrators, microcolumns and microsensors. $\mu$-GC systems allow the separation of target compounds in highly reduced times when compared with conventional GC. The reduced power consumption is another benefit derived from the miniaturization of these systems, since the energy requirements to heat and cool smaller individual components are decreased (Dorman et al., 2010). Apart from its application in medical analysis, on-site gaseous sampling and analysis can be performed by portable and microfabricated GC, thus minimizing the problems related to analyte losses (mainly due to adsorption and reactions) that can be produced during sampling and transport (Ohira \& Toda, 2008).

\subsubsection{Liquid Chromatography}

LC is an essential separation technique for a large variety of scientific areas. The miniaturization of LC has been mainly derived from the reduction of the analytical column dimensions, the development of novel stationary phase materials and the improvement of detection systems.

The decrease in the internal diameter of the analytical columns for the achievement of efficient separations was first proposed by Ishii et al. (1977) and from this innovative work, great efforts have been made to achieve a high degree of miniaturization of these analytical separation systems, yielding micro-LC, capillary LC, nano-LC and chip-based LC. The reduction of the dimensions of the LC column has an important influence on the remaining components of the chromatographic system, including the connecting tubing, injector, and detection cell volume and shape. Specifically, the tubing used to connect the LC column with the injector and the detector should be downscaled in accordance with the decrease of the chromatographic column dimensions, or even removed when the internal diameter of the LC column is lower than 100 $\mu \mathrm{m}$. In addition, a reduction of the internal diameter of the chromatographic column can give rise to a decreased sensitivity that could be counteracted by improving the geometry of the detection system (Szumski \& Buszewski, 2002).

The development of novel stationary phase materials with improved mechanical strength and chemical inertness, as well as enhanced surface area-to-volume ratio has also enabled the miniaturization of LC systems. The separation performance is, however, not just dependent on the particle size, but also on parameters such as monodispersity or porosity (Kutter, 2012). The reduction of the particle diameter of 
A)

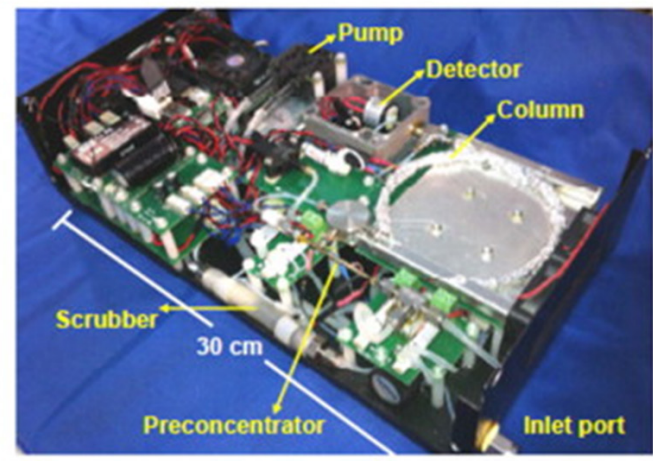

B)

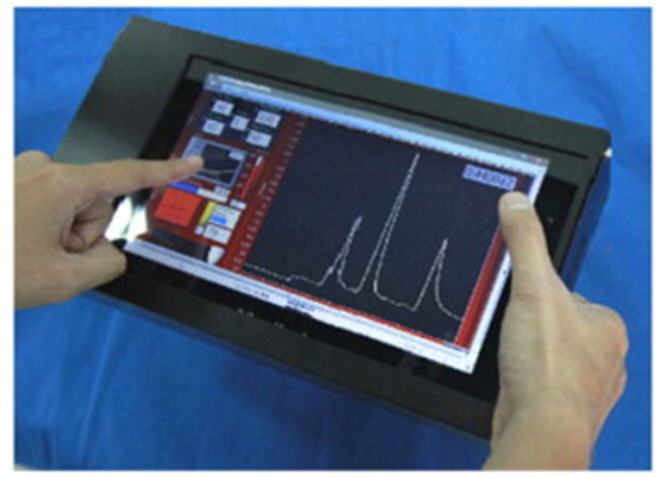

Figure 1.8 Photographs of prototype $\mu$-GC: (A) interior structure with top lid and computer removed; and (B) complete system with built-in tablet computer. Reprinted from Jian et al. (2013) with permission from Elsevier.

the packing material of LC columns has been proposed with the aim of achieving higher resolution with reduced analysis times, even though extreme pressures are a requirement for optimal performance (Jerkovich et al., 2003). Capillary electrochromatography and ultra-high pressure LC techniques have been derived from the use of LC columns with micrometer-sized particles to overcome pressure limitations.

The sensitivity is compromised when on-column detection is performed as a result of the reduction of the analytical dimensions of the LC columns and the reduced sample volumes injected. Nevertheless, it is worth noting that electrospray ionization-mass spectrometry (ESI-MS) performs optimally at very low flow rates, thus having excellent suitability and compatibility with a miniaturized LC column (Desmet \& Eeltink, 2013). Furthermore, certain strategies have been applied to enhance the sensitivity when miniaturized LC columns were used, for example selection of detection systems with improved geometry, on-column focusing, and on-line and off-line preconcentration (Zotou, 2012).

Chip-based LC systems for the separation of target analytes in a channel of a chip device were first reported in 1990 (Manz et al., 1990b). The proposed system involved a 5 x $5 \mathrm{~mm}$ silicon chip containing an open-tubular column of $6 \mu \mathrm{m} \times 2 \mu \mathrm{m} \times 15 \mathrm{~cm}$ 
and a conductometric detector. Since this first work, many chip-based LC systems have been reported in the literature, with applicability in environmental and clinical analysis, proteomics and genetics, among other areas. The improvement of chipbased LC systems is linked to the development of novel stationary phase materials. The separation efficiency is highly dependent on the channel size, shape and aspect ratio in microchip-based LC systems (Kutter, 2012). The improvements experienced in machining methods allow the highly accurate preparation of microchannels on the surface of silicon glass or polymeric planar substrates.

\subsubsection{Capillary Electrophoresis}

$\mathrm{CE}$ is a versatile analytical separation technique which provides high separation efficiency, short analysis times and reduced consumption of sample and electrolyte. The employment of $75 \mu \mathrm{m}$ internal diameter open-tubular glass capillaries in zone electrophoresis was firstly proposed by Jorgensen and Luckacs (1981). The reduction of the inner diameter of the capillary involved an efficient dissipation of heat produced at high voltages, thus yielding highly efficient separations.

Both capillary-based CE and chip-based CE have been proposed in the literature to enable the miniaturization and portability of commercially available electrophoresis instrumentation. Capillary-based CE systems (also referred in the literature as non-chip based CE) are fabricated around a cylindrical capillary, while microfluidic channels are fabricated in chip-based CE systems by means of microfabrication techniques (Lewis et al., 2013). CE is highly amenable to portability due to it is relative simplicity as well as advantages such as the low consumption of buffer solution, few moving parts and, unlike LC systems, the absence of a pump which is not a requirement in CE.

In 1998, Kappes and Hauser (1998) presented the first portable CE instrument. The proposed instrument included, among other components, a $25 \mu \mathrm{m}$ inner diameter fused-silica capillary, a potentiometric detector and two $12 \mathrm{~V}$ lead-acid batteries. The samples were electrokinetically injected. The whole CE instrument was contained in a PVC case of dimensions $340 \times 175 \times 175 \mathrm{~mm}$ and had a total weight of $7.5 \mathrm{~kg}$. The applicability of the proposed field-portable CE instrument was expanded by using other two electrochemical detection techniques, namely amperometry and conductometry (Kappes et al., 2001).

Harrison et al. presented the first chip-based CE system (Harrison et al., 1992). The development of micromachining techniques allowed the formation of capillary channels in a planar glass substrate. The separation efficiency of the system was comparable to conventional open tubular capillaries when expressed as number of plates per volt. Field-portable chip-based CE systems have been reported in the literature with improved dimensions and weight, mainly due to the incorporation of small high-voltage power supplies and on-chip detectors. For instance, the first portable chip-based CE system showed highly reduced dimensions (102 x 152 x $25 \mathrm{~mm})$ and weight $(0.35 \mathrm{~kg})$ 
(Jackson et al., 2003). Two recent reviews dealing with the development of portable CE can be consulted for further details (Ryvolová et al., 2010; Lewis et al., 2013).

Novel developments towards the fabrication of autonomous chip-based CE systems for remote analysis have been recently reported. These systems allow one to perform every step of the analytical process (sampling, sample preparation, separation and detection) without the participation of the user. Culbertson et al. presented a portable, battery-operated microfluidic system capable of performing electrophoretic separations in less than $12 \mathrm{~s}$ under microgravity and hypergravity conditions (Culbertson et al., 2005). Skelley et al. presented a microfabricated CE instrument called the Mars Organic Analyzer (MOA) capable of determining key biomarkers present in Mars-like soil samples (Skelley et al., 2005). The MOA showed a weight of $11 \mathrm{~kg}$ and a peak power utilization of $15 \mathrm{~W}$. The concept of lab-on-a-robot was introduced in 2008 by Berg et al. to carry out remote wireless analysis (Berg et al., 2008). This system consisted of the combination of a chip-based CE system, a mobile platform and wireless global position system (GPS) (Figure 1.9). The miniaturized system allows the user to select the sampling point by means of the GPS and transmitted video, followed by the collection of a gas sample, injection, separation, detection, and transfer of the data to a distant control unit.

Certain advantages are attributed to chip-based CE systems. Apart from the obvious reduction of the instrumentation size, low consumption of sample and chemicals, lower power requirements and expeditious separations are among the benefits of chip-based CE. However, it has been reported that quantitative analysis with microchip CE can be difficult for non-experienced operators, since several factors can affect the accuracy and repeatability, such as buffer electrolysis, bubble formation and clogging events, analyte-surface interactions, as well as injection and power supply-related problems (Revermann et al., 2008). Furthermore, the use of emerging materials in the fabrication of the channel surface of chip-based CE can hinder the prediction of the electroosmotic flow characteristics since the electroosmotic flow rate depends on the channel surface characteristics (Lewis et al., 2013).

It is interesting to note that the development of chip-based systems has employed mainly electrophoresis as the separation technique of choice. In general, CE is considered to be inferior to LC owing to its lack of robustness (Breadmore, 2012). Nevertheless, CE is more amenable to microchips than LC, so the current trend of miniaturization in analytical chemistry may afford new opportunities to electrophoresis.

\subsubsection{Miniaturization of Detection Techniques}

The advances achieved in fields such as electronics, engineering and material sciences have allowed the miniaturization of analytical detection systems. The characteristic features of MEMS fabrication techniques, namely miniaturization, multiplicity and microelectronics, have enabled the batch production of small-sized detection 


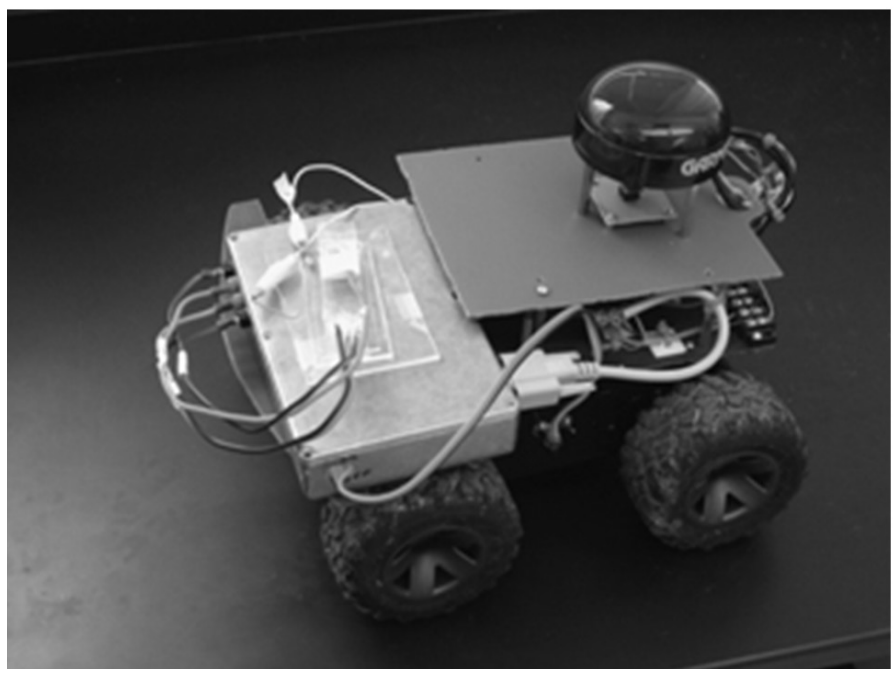

Figure 1.9 Picture of the assembled lab-on-a-robot containing high-voltage power supply, electrochemical detector, mobile platform, field programmable gate array, radio frequency modem, compass, and GPS. Reprinted from Berg et al. (2008) with permission from John Wiley \& Sons.

systems (Schuler et al., 2009). Miniaturization of analytical detection techniques requires the size reduction of different elements of conventionally-sized instruments without ignoring the performance of each component, in such a way that the overall miniaturized detection techniques yield comparable or improved analytical performance. Scale-down analytical instrumentation has been accompanied by the required advances on auxiliary devices, including micropumps (Chen et al., 2008), microvalves (Oh \& Ahn, 2006), micromixers (Nguyen \& Wu, 2005). It is also worth mentioning that the miniaturization of analytical instrumentation is dependent on the size of electronic systems. The advances towards the miniaturization of power sources and their integration into electronic subsystems have allowed the development of microbatteries with a size at least 500-fold smaller than commercially available batteries (Heller, 2006). In this section, a brief description of miniaturized detection systems, such as molecular and atomic spectrometry, mass spectrometry and electrochemical techniques, is discussed.

\subsubsection{Molecular Spectrometry}

Ultraviolet and visible (UV-Vis) absorption spectrometry is widely used due to its simplicity, rapidity and low cost. Decreases in the size of the sample compartment, together with improvements in radiation sources, wavelength discrimination components, fiber optics technology and the implementation of detector arrays have allowed the downscale of conventionally-sized UV-Vis spectrometers. Analysis of microliter to nanoliter sample volumes, increased optical path length-to-sample volume ratio, 
rapidity, low power requirements and portability are the main advantages obtained by miniaturizing UV-Vis spectrophotometers. The loss of sensitivity is, however, a drawback that can be alleviated or even resolved by increasing the optical path length by axial-direction, multireflection, liquid-core waveguides or cavity ring down spectroscopy, or by combining the different microvolume UV-Vis spectrophotometric systems available with miniaturized sample preparation techniques for appropriate preconcentration prior to the analysis. Some reviews on the miniaturization of UV-Vis spectrometric systems and accessories are available (Bacon et al., 2004; Pena-Pereira et al., 2011).

Technological improvements towards miniaturized infrared (IR) spectroscopic systems have also been reported. Specifically, the main improvements have been focused on the light sources, the interferometer and electronic circuits. A significant improvement concerning light sources for IR spectroscopy has been achieved with the development of quantum cascade lasers (QCL) (Faist et al., 1994). QCLs are characterized by their large emission power, reliability, wavelength tenability and lifetime (Kim et al., 2008). The developments achieved have enabled the hyphenation of analytical separation techniques such as LC and CE with IR spectroscopy (Kuligowski et al., 2010).

\subsubsection{Atomic Spectrometry}

Recent efforts towards shrinking plasma sources have advanced the miniaturization of analytical atomic spectrometric techniques. Plasmas consist on a highly energized mixture of positive ions and their electrons in a neutral background gas. They are initiated by supplying energy to a volume that contains a neutral gas. The miniaturization of plasma sources has emerged in response to the necessity of small and lightweight instrumentation with the potential for portability, improved cost-to-performance ratio, rapidity, reduced power and gas consumption, as well as integration with separation and detection techniques.

A variety of miniaturized plasma sources can be produced by the application of direct current (dc), alternating current (ac), radio frequency and microwave power. Those plasmas confined to dimensions of $1 \mathrm{~mm}$ or less are defined as “microplasmas". When coupled with optical or mass spectrometry, microplasmas are powerful tools for the detection of molecular fragments and elemental analysis. Most of the microplasmas reported in the literature have been applied to the analysis of gases or liquid samples using volatilization reactions prior to analysis. In fact, it has been stated that microplasmas lack stability in the presence of significant solvent loads more than fullsized plasmas, probably due to the combination of shorter residence times and lower gas temperatures (Webb et al., 2007). Nevertheless, some advances allow the analysis of liquid phases using microplasmas as emission sources. For instance, Webb et al. employed a small-scale ( $5 \times 2 \mathrm{~mm}$ ) atmospheric-pressure helium discharge that was demonstrated to be stable in the presence of significant solvent loads. The particular 
geometry of the source has been identified as the factor that mainly contributed to its robustness (Webb et al., 2007). Staack et al. reported the generation of a plasma discharge around electrodes with ultrasharp tips and elongated nanoparticles that enable the simultaneous determination of dissolved elements present in liquid matrices by optical emission spectroscopy (Staack et al., 2008).

The advances achieved in microfabrication techniques have enabled the integration of microplasma sources with LOC systems. The possibility of operating at atmospheric pressure affords an important advantage towards the integration of microplasmas in analytical microsystems and the development of portable devices since vacuum pumps are not required (Luo \& Duan, 2012). It is also noteworthy to mention that microplasma-based atomic emission detectors have been coupled with GC for analytical purposes (Miclea et al., 2007).

The development and application of microplasmas has been discussed in a number of excellent reviews (Broekaert, 2002; Franzke et al., 2003; Karanassios, 2004; Miclea \& Franzke, 2007; Luo \& Duan, 2012) to which interested readers are referred for further details.

\subsubsection{Mass Spectrometry}

Mass spectrometry is a powerful analytical technique due to its high sensitivity, selectivity and speed, being widely used for both qualitative and quantitative analysis on the basis of the mass-to-charge ratio of ionized atoms and molecules. A mass spectrometer comprises three essential parts, namely the ionization source, mass selective analyzer and ion detector. Furthermore, a vacuum system and a control system are important parts of a mass spectrometer. The miniaturization of mass spectrometers has focused on downsizing mass selective analyzers. The miniaturization of a variety of mass analyzers has been reported in the literature, including miniature quadrupole, magnetic sector, time-of-flight (TOF), quadrupole ion trap and ion cyclotron resonance (Henry, 1999; Ouyang \& Cooks, 2009) detectors. However, depending on the type of mass analyzer, the size reduction can be accompanied with a loss of mass resolution which therefore affects the mass analysis. Furthermore, the size reduction of the mass analyzer can also show an impact on both vacuum and control systems (Ouyang \& Cooks, 2009). Developments in the design of vacuum systems and the improvement of the control system by means of modern electronics have been key milestones towards miniaturized mass spectrometers with reduced weight, size and power consumption. The vacuum system is commonly the heaviest and most powerconsuming part of a mass spectrometer. Even though miniature mass spectrometers do not require pressures as low as the ones used in conventional spectrometers, the design of the vacuum system highly influences the performance of the mass spectrometer. Miniaturized high vacuum pumps have been reported in the literature, including turbo-molecular pumps, cryogenic pumps and ion-getter pumps, the former being the only reliable option when dealing with high vacuum pumping. Ion-getter pumps 
only operate below $10^{-3}$ torr, in such a way that a pre-vacuum pump is required with portable systems that operate with ion-getter pumps when starting from atmospheric pressure (Ouyang \& Cooks, 2009). Nevertheless, the use of ion-getter pumps in combination with an auxiliary pump has been a common strategy that allowed the development of portable mass spectrometers. Yang et al. reported the development of a palm portable mass spectrometer for the determination of chemical warfare agents in the field (Yang et al., 2008). The miniaturized instrument, with a volume of $1.54 \mathrm{~L}$ and a weight of $1.48 \mathrm{~kg}$, employed a miniaturized ion trap as the mass analyzer, an ion-getter pump and an embedded microcomputer for system control, all operating on a battery power of $5 \mathrm{~W}$.

The miniaturization of the ion source has been mainly focused on ESI, even though other atmospheric pressure ion sources such as atmospheric pressure chemical ionization, atmospheric pressure photoionization and electron ionization have been reported in the literature (Sikanen et al., 2010). The miniaturized ESI, named as nano-electrospray ion source, was first reported by Wilm and Mann (1996). ESI is easily downscaled and offers high sensitivity at low flow rates (nL $\min ^{-1}$ ) using extremely small sample volumes. Moreover, ESI-MS can be easily interfaced with microfluidic systems, LC and CE.

\subsubsection{Electrochemical Techniques}

Electrochemical techniques have been employed in several scientific areas due to their high sensitivity, ease of operation, portability and low cost. Several miniaturized electrochemical techniques are commonly employed. The most representative example is the hand-held glucose meter for insulin-dependent diabetes which operates using amperometric enzyme electrodes (Wang, 2002). Furthermore, a variety of sensors have been reported for the determination of a wide range of target compounds, as has been outlined in two excellent review articles (Stetter, 2008; Kimmel et al., 2012).

Electrochemical detection techniques are well-suited to miniaturization, since they can be produced by conventional microfabrication methods and their analytical performance is not affected by the decrease in size, enabling sensitive detection of electroactive target compounds in picoliter to nanoliter sample volumes. Thus, electrochemical detectors, including voltammetric, conductometric and potentiometric detectors, have been implemented in LOC systems (Nyholm, 2005).

The development of small-size voltammetric working electrodes was reported for the first time in 1981 by Wightman who was interested in identifying changes in the chemical concentrations of neurotransmitters inside the mammalian brain (Wightman, 1981). In 1990, Penner et al. reported the fabrication of nanometer-scale electrodes that enabled the determination of electron transfer rate constants two orders of magnitude faster than with conventional electrochemical methods (Penner et al., 1990). Voltammetric electrodes with at least one dimension below $10 \mu \mathrm{m}$ are known 
as "microelectrodes" (also reported as ultramicroelectrodes), while the term "nanoelectrodes" typically refers to voltammetric electrodes with at least one dimension below $100 \mathrm{~nm}$. Thus, nanoelectrodes can be considered as a special type of microelectrode. Both microelectrodes and nanoelectrodes have been extensively employed in the literature, enabling remarkable advances in both fundamental and applied electrochemical research, such as electroanalysis in single cells and biomolecules, as well as electrocatalysis at nano-scales (Li \& Hu, 2013; Oja et al., 2013).

Among the advantages of reducing the geometrical dimensions of voltammetric electrodes are the decrease of the ohmic drop potential, the fast establishment of a steady-state signal, current increase due to the enhanced mass transport, and the increase of the signal-to-noise ratio achieved in small size electrodes (Štulík et al., 2000). Furthermore, unlike conventional-sized electrodes (of few mm or larger), ultra small electrodes can be employed on samples of very high resistance, such as solutions without added supporting electrolyte. This is a consequence of the extremely small currents at these electrodes. Moreover, the reduced size of the electrodes enables the analysis of extremely small volumes of sample, that can be down to the attoliter level. This fact is especially relevant in areas of bioanalysis and medicine, for example, in the detection and identification of neurotransmitters and other types of chemical messengers released from certain types of cells ( $\mathrm{Li} \& \mathrm{Hu}, 2011$ ).

Small size electrodes with several geometries have been reported in the literature, including micro- and nanodisks, cylinders, arrays, bands, rings, spheres, hemispheres, and more recently, nanopores. Among the different geometries, the microdisk electrode is the most commonly employed ( $\mathrm{Li} \& \mathrm{Hu}, 2013$ ).

A variety of challenging areas have progressed substantially in recent years thanks to the development of voltammetric micro and nanoelectrodes, for example single molecule detection, single nanoparticle electrochemistry and the determination of neurotransmitters from single neuronal cells (Cox \& Zhang, 2012).

\subsection{Conclusions and Outlook}

The motivating factors behind the miniaturization of analytical systems are diverse. First of all, we can consider the downscale of analytical instrumentation as a natural means to get more space in the laboratory. The size decrease on analytical instrumentation has been evident during the last few decades as a result of technological improvements. Nevertheless, the miniaturization of analytical systems today is more focused on the improvement of their analytical performance, rapidity, environmental sustainability and economic benefits than simply on reducing their size. In these terms, the miniaturization of the different steps of the analytical process proved to be advantageous in comparison with conventional analytical systems. However, it should not be forgotten that significant drawbacks can be produced when dealing with samples below microliter volume scales. Thus, in certain cases, the development 
of a miniaturized alternative to conventional analytical methods shows certain limitations that result in novel problems to solve.

The development of portable and eventually handheld instrumentation for field analysis has also been boosted by the improvements in miniaturization. Furthermore, the novel requirements of challenging research areas and applications, including but not limited to proteomics, metabolomics, forensics or remote sampling have allowed further improvements on miniaturized analytical systems. Shrinking analytical systems are continuously being improved by scientific and technological developments, including novel materials, fabrication techniques, advanced auxiliary components and improved designs. Therefore, further efforts can be envisaged on miniaturization, portability and intelligentization of analytical systems in the near future.

\section{Abbreviations}

$\begin{array}{ll}\mu \text {-GC } & \text { micro gas chromatograph } \\ \mu \text { SI } & \text { micro-sequential injection } \\ \mu \text {-TAS } & \text { micro-total analysis systems } \\ \text { CE } & \text { capillary electrophoresis } \\ \text { DLLME } & \text { dispersive liquid-liquid microextraction } \\ \text { EE } & \text { extraction efficiency } \\ \text { EF } & \text { enrichment factor } \\ \text { ESI } & \text { electrospray ionization } \\ \text { GC } & \text { gas chromatography } \\ \text { GPS } & \text { global position system } \\ \text { IR } & \text { infrared } \\ \text { LC } & \text { liquid chromatography } \\ \text { LOC } & \text { lab-on-a-chip } \\ \text { LOV } & \text { lab-on-a-valve } \\ \text { LPME } & \text { liquid-phase microextraction } \\ \text { MEMS } & \text { micro-electromechanical systems } \\ \text { MOA } & \text { Mars Organic Analyzer } \\ \text { MS } & \text { mass spectrometry } \\ \text { QCL } & \text { quantum cascade laser } \\ \text { SBSE } & \text { stir bar sorptive extraction } \\ \text { SDME } & \text { single-drop microextraction } \\ \text { SPE } & \text { solid-phase extraction } \\ \text { SPME } & \text { solid-phase microextraction } \\ \text { TAS } & \text { total analysis systems } \\ \text { TMS } & \text { toroidal ion trap mass spectrometer } \\ \text { TOF } & \text { time of flight } \\ \text { UV-Vis } & \text { ultraviolet and visible. } \\ & \end{array}$




\section{Acknowledgements}

F. Pena-Pereira thanks Xunta de Galicia for financial support as a post-doctoral researcher of the $\mathrm{I} 2 \mathrm{C}$ program.

\section{References}

Arthur, C. L., \& Pawliszyn, J. (1990). Solid phase microextraction with thermal desorption using fused silica optical fibers. Analytical Chemistry, 62, 2145-2148.

Bacon, C. P., Mattley, Y., \& DeFrece, R. (2004). Miniature spectroscopic instrumentation: applications to biology and chemistry. Review of Scientific Instruments, 75, 1-16.

Baltussen, E., Sandra, P., David, F., \& Cramers, C. (1999). Stir bar sorptive extraction (SBSE), a novel extraction technique for aqueous samples: theory and principles. Journal of Microcolumn Separations, 11, 737-747.

Bartle, K. D., \& Myers, P. (2002). History of gas chromatography. TrAC Trends in Analytical Chemistry, 21, 547-557.

Berg, C., Valdez, D. C., Bergeron, P., Mora, M. F., Garcia, C. D., \& Ayon, A. (2008). Lab-on-a-robot: integrated microchip CE, power supply, electrochemical detector, wireless unit, and mobile platform. Electrophoresis, 29, 4914-4921.

Breadmore, M. C. (2012). Capillary and microchip electrophoresis: challenging the common conceptions. Journal of Chromatography A, 1221, 42-55.

Broekaert, J. A. (2002). The development of microplasmas for spectrochemical analysis. Analytical and Bioanalytical Chemistry, 374, 182-187.

Capitan-Vallvey, L. F., \& Palma, A. J. (2011). Recent developments in handheld and portable optosensing-a review. Analytica Chimica Acta, 696, 27-46.

Chen, L., Lee, S., Choo, J., \& Lee, E. K. (2008). Continuous dynamic flow micropumps for microfluid manipulation. Journal of Micromechanics and Microengineering, 18, 013001.

Contreras, J. A., Murray, J. A., Tolley, S. E., Oliphant, J. L., Tolley, H. D., Lammert, S. A., et al. (2008). Hand-portable gas chromatograph-toroidal ion trap mass spectrometer (GC-TMS) for detection of hazardous compounds. Journal of the American Society for Mass Spectrometry, 19, 1425-1434.

Cox, J. T., \& Zhang, B. (2012). Nanoelectrodes: recent advances and new directions. Annual Review of Analytical Chemistry, 5, 253-272.

Culbertson, C. T., Tugnawat, Y., Meyer, A. R., Roman, G. T., Ramsey, J. M., \& Gonda, S. R. (2005). Microchip separations in reduced-gravity and hypergravity environments. Analytical Chemistry, 77, 7933-7940.

Desmet, G., \& Eeltink, S. (2013). Fundamentals for LC miniaturization. Analytical Chemistry, 85, 543-556.

Dorman, F. L., Whiting, J. J., Cochran, J. W., \& Gardea-Torresdey, J. (2010). Gas chromatography. Analytical Chemistry, 82, 4775-4785.

Faist, J., Capasso, F., Sivco, D. L., Sirtori, C., Hutchinson, A. L., \& Cho, A. Y. (1994). Quantum cascade laser. Science, 264, 553-556.

Feynman, R. P. (1992). There's plenty of room at the bottom. Journal of Microelectromechanical Systems, 1, 60-66.

Franzke, J., Kunze, K., Miclea, M., \& Niemax, K. (2003). Microplasmas for analytical spectrometry. Journal of Analytical Atomic Spectrometry, 18, 802-807. 
Garrigues, S., Armenta, S., \& de la Guardia, M. (2010). Green strategies for decontamination of analytical wastes. TrAC Trends in Analytical Chemistry, 29, 592-601.

Golay, M. J. E. (1958). In D. H. Desty (Ed.), Gas Chromatography (pp. 36-55). London: Butterworths.

Hansen, E. H., \& Wang, J. (2005). The three generations of flow injection analysis. Analytical Letters, 37, 345-359.

Harrison, D. J., Manz, A., Fan, Z., Lüdi, H., \& Widmer, M. (1992). Capillary electrophoresis and sample injection systems integrated on a planar glass chip. Analytical Chemistry, 64, 1928-1932.

Heller, A. (2006). Potentially implantable miniature batteries. Analytical and Bioanalytical Chemistry, 385, 469-473.

Henry, C. M. (1999). The incredible shrinking mass spectrometers. Analytical Chemistry, 71, 264A-268A.

Herold, K. E., \& Rasooly, A. (2009). Lab on a Chip Technology (Vol. 1): Fabrication and Microfluidics. Norfolk, UK.: Caister Academic Press.

Idris, A. M. (2010). An overview of the generations and recent versions of flow injection techniques. Critical Reviews in Analytical Chemistry, 40, 150-158.

Ishii, D., Asai, K., Hibi, K., Onokuchi, T., \& Nagaya, M. (1977). A study of micro-high-performance liquid chromatography: I. Development of technique for miniaturization of high-performance liquid chromatography. Journal of Chromatography A, 144, 157-168.

Jackson, D. J., Naber, J. F., Roussel Jr., T. J., Crain, M. M., Walsh, K. M., Keynton, R. S., et al. (2003). Portable high-voltage power supply and electrochemical detection circuits for microchip capillary electrophoresis. Analytical Chemistry, 75, 3643-33649.

James, A., \& Martin, A. (1952). Gas-liquid partition chromatography: the separation and microestimation of volatile fatty acids from formic acid to dodecanoic acid. Biochemical Journal, 50, 679-690.

Jeannot, M. A., \& Cantwell, F. F. (1996). Solvent microextraction into a single drop. Analytical Chemistry, 68, 2236-2240.

Jerkovich, A. D., Mellors, J. S., \& Jorgenson, J. W. (2003). The use of micrometer-sized particles in ultrahigh pressure liquid chromatography. LCGC North America, 21, 600-610.

Jian, R.-S., Huang, Y.-S., Lai, S.-L., Sung, L.-Y., \& Lu, C.-J. (2013). Compact instrumentation of a $\mu$-GC for real time analysis of sub-ppb VOC mixtures. Microchemical Journal, 108, 161-167.

Jorgenson, J. W., \& Lukacs, K. D. (1981). Zone electrophoresis in open-tubular glass capillaries. Analytical Chemistry, 53, 1298-1302.

Kappes, T., Galliker, B., Schwarz, M. A., \& Hauser, P. C. (2001). Portable capillary electrophoresis instrument with amperometric, potentiometric and conductometric detection. TrAC Trends in Analytical Chemistry, 20, 133-139.

Kappes, T., \& Hauser, P. C. (1998). Portable capillary electrophoresis instrument with potentiometric detection. Analytical Communications, 35, 325-329.

Karanassios, V. (2004). Microplasmas for chemical analysis: analytical tools or research toys? Spectrochimica Acta Part B: Atomic Spectroscopy, 59(7), 909-928.

Kim, S. S., Young, C., \& Mizaikoff, B. (2008). Miniaturized mid-infrared sensor technologies. Analytical and Bioanalytical Chemistry, 390, 231-237.

Kimmel, D. W., LeBlanc, G., Meschievitz, M. E., \& Cliffel, D. E. (2012). Electrochemical sensors and biosensors. Analytical Chemistry, 84, 685-707.

Kuligowski, J., Quintas, G., de la Guardia, M., \& Lendl, B. (2010). Analytical potential of mid-infrared detection in capillary electrophoresis and liquid chromatography: a review. Analytica Chimica Acta, 679, 31-42.

Kutter, J. P. (2012). Liquid phase chromatography on microchips. Journal of Chromatography A, 1221, 72-82. 
Lewis, A. P., Cranny, A., Harris, N. R., Green, N. G., Wharton, J. A., Wood, R. J. K., et al. (2013). Review on the development of truly portable and in-situ capillary electrophoresis systems. Measurement Science and Technology, 24, 042001.

Li, C. M., \& Hu, W. (2013). Electroanalysis in micro- and nano-scales. Journal of Electroanalytical Chemistry, 688, 20-31.

Li, T., \& Hu, W. (2011). Electrochemistry in nanoscopic volumes. Nanoscale, 3, 166-176.

Lin, B. (2011). Microfluidics: Techologies and applications. Heidelberg, Germany: Springer.

Liu, S., \& Dasgupta, P. K. (1995). Liquid droplet. A renewable gas sampling interface. Analytical Chemistry, 67, 2042-2049.

Livak-Dahl, E., Sinn, I., \& Burns, M. (2011). Microfluidic chemical analysis systems. Annual Review of Chemical and Biomolecular Engineering, 2, 325-353.

Luo, D., \& Duan, Y. (2012). Microplasmas for analytical applications of lab-on-a-chip. TrAC Trends in Analytical Chemistry, 39, 254-266.

Luque de Castro, M. D., \& Priego Capote, F. (2008). Miniaturisation of analytical steps: necessity and snobbism. Analytical and Bioanalytical Chemistry, 390, 67-69.

Manz, A., Graber, N., \& Widmer, H. M. (1990a). Miniaturized total chemical analysis systems: a novel concept for chemical sensing. Sensors and Actuators B, 1, 244-248.

Manz, A., Miyahara, Y., Miura, J., Watanabe, Y., Miyagi, H., \& Sato, K. (1990b). Design of an open-tubular column liquid chromatograph using silicon chip technology. Sensors and Actuators B, 1, 249-255.

Mark, D., Haeberle, S., Roth, G., von Stetten, F., \& Zengerle, R. (2010). Microfluidic lab-on-a-chip platforms: requirements, characteristics and applications. Chemical Society Reviews, 39, 1153-1182.

Marx, A., Fettinger, J., Verpoorte, E., Liidi, H., Widmer, H., \& Harrison, D. (1991). Micromachining of monocrystalline silicon and glass for chemical analysis systems A look into next century's technology or just a fashionable craze? TrAC Trends in Analytical Chemistry, 10, 144-149.

Miclea, M., \& Franzke, J. (2007). Analytical detectors based on microplasma spectrometry. Plasma Chemistry and Plasma Processing, 27, 205-224.

Miclea, M., Okruss, M., Kunze, K., Ahlman, N., \& Franzke, J. (2007). Microplasma-based atomic emission detectors for gas chromatography. Analytical and Bioanalytical Chemistry, 388, 1565-1572.

Mittermuller, M., \& Volmer, D. A. (2012). Micro- and nanostructures and their application in gas chromatography. The Analyst, 137, 3195-3201.

Nge, P. N., Rogers, C. I., \& Woolley, A. T. (2013). Advances in microfluidic materials, functions, integration, and applications. Chemical Reviews, 113, 2550-2583.

Nguyen, N.-T., \& Wu, Z. (2005). Micromixers-a review. Journal of Micromechanics and Microengineering, 15, R1-R16.

Nyholm, L. (2005). Electrochemical techniques for lab-on-a-chip applications. The Analyst, 130, 599-605.

Oh, K. W., \& Ahn, C. H. (2006). A review of microvalves. Journal of Micromechanics and Microengineering, 16, R13-R39.

Ohira, S., \& Toda, K. (2008). Micro gas analyzers for environmental and medical applications. Analytica Chimica Acta, 619, 143-156.

Oja, S. M., Wood, M., \& Zhang, B. (2013). Nanoscale electrochemistry. Analytical Chemistry, 85, 473-486.

Ouyang, Z., \& Cooks, R. G. (2009). Miniature mass spectrometers. Annual Review of Analytical Chemistry, 2, 187-214.

Pedersen-Bjergaard, S., \& Rasmussen, K. E. (1999). Liquid-liquid-liquid microextraction for sample preparation of biological fluids prior to capillary electrophoresis. Analytical Chemistry, 71, 2650-2656. 
Pena-Pereira, F., Costas-Mora, I., Romero, V., Lavilla, I., \& Bendicho, C. (2011). Advances in miniaturized UV-Vis spectrometric systems. TrAC Trends in Analytical Chemistry, 30, 1637-1648.

Penner, R. M., Heben, M. J., Longin, T. L., \& Lewis, N. S. (1990). Fabrication and use of nanometersized electrodes in electrochemistry. Science, 250, 1118-1121.

Priego-Capote, F., \& de Castro, L. (2006). Ultrasound-assisted levitation: Lab-on-a-drop. TrAC Trends in Analytical Chemistry, 25, 856-867.

Radadia, A., Salehi-Khojin, A., Masela, R., \& Shannon, M. (2010). The effect of microcolumn geometry on the performance of micro-gas chromatography columns for chip scale gas analyzers. Sensors and Actuators B, 150, 456-464.

Revermann, T., Gotz, S., Kunnemeyer, J., \& Karst, U. (2008). Quantitative analysis by microchip capillary electrophoresis: current limitations and problem-solving strategies. The Analyst, 133, 167-174.

Rezaee, M., Assadi, Y., Milani Hosseini, M.-R., Aghaee, E., Ahmadi, F., \& Berijani, S. (2006). Determination of organic compounds in water using dispersive liquid-liquid microextraction. Journal of Chromatography A, 1116, 1-9.

Ríos, A., Escarpa, A., \& Simonet, B. (2009). Miniaturization of analytical systems: Principles, designs and applications. Chichester, UK: Wiley.

Rios, A., Zougagh, M., \& Avila, M. (2012). Miniaturization through lab-on-a-chip: utopia or reality for routine laboratories? A review. Analytica Chimica Acta, 740,1-11.

Ruzicka, J. (2000). Lab-on-valve: universal microflow analyzer based on sequential and bead injection. The Analyst, 125, 1053-1060.

Ryvolová, M., Macka, M., \& Preisler, J. (2010). Portable capillary-based (non-chip) capillary electrophoresis. TrAC Trends in Analytical Chemistry, 29, 339-353.

Santesson, S., \& Nilsson, S. (2004). Airborne chemistry: acoustic levitation in chemical analysis. Analytical and Bioanalytical Chemistry, 378, 1704-1709.

Schuler, L. P., Milne, J. S., Dell, J. M., \& Faraone, L. (2009). MEMS-based microspectrometer technologies for NIR and MIR wavelengths. Journal of Physics D: Applied Physics, 42, 133001.

Senzel, A. (1970). Miniaturization in analytical instrumentation. Analytical Chemistry, 42, 79A-81A.

Sikanen, T., Franssila, S., Kauppila, T. J., Kostiainen, R., Kotiaho, T., \& Ketola, R. A. (2010). Microchip technology in mass spectrometry. Mass Spectrometry Reviews, 29, 351-391.

Skelley, A. M., Scherer, J. R., Aubrey, A. D., Grover, W. H., Ivester, R. H., Ehrenfreund, P., et al. (2005). Development and evaluation of a microdevice for amino acid biomarker detection and analysis on Mars. Proceedings of the National Academy of Sciences of the United States of America, 102, 1041-1046.

Staack, D., Fridman, A., Gutsol, A., Gogotsi, Y., \& Friedman, G. (2008). Nanoscale corona discharge in liquids, enabling nanosecond optical emission spectroscopy. Angewandte Chemie International Edition, 47, 8020-8024.

Stetter, J. R. (2008). Amperometric gas sensors - A review. Chemical Reviews, 108, 352-366.

Štulík, K., Amatore, C., Holub, K., Mareček, V., \& Kutner, W. (2000). Microelectrodes. Definitions, characterization, and applications. Pure and Applied Chemistry, 72, 1483-1492.

Szumski, M., \& Buszewski, B. (2002). State of the art in miniaturized separation techniques. Critical Reviews in Analytical Chemistry, 32, 1-46.

Terry, S. C., Jerman, J. H., \& Angell, J. B. (1979). A gas chromatographic air analyzer fabricated on a silicon wafer. IEEE Transactions on Electronic devices, 26, 1880-1886.

Wang, J. (2002). Portable electrochemical systems. TrAC Trends in Analytical Chemistry, 21, 226-232.

Webb, M. R., Andrade, F. J., \& Hieftje, G. M. (2007). The annular glow discharge: a small-scale plasma for solution analysis. Journal of Analytical Atomic Spectrometry, 22, 775-782.

Wightman, R. M. (1981). Microvoltammetric electrodes. Analytical Chemistry, 53, 1125A-1134A.

Wilm, M., \& Mann, M. (1996). Analytical properties of the nanoelectrospray ion source. Analytical Chemistry, 68, 1-8. 
Yang, M., Kim, T. Y., Hwang, H. C., Yi, S. K., \& Kim, D. H. (2008). Development of a palm portable mass spectrometer. Journal of the American Society for Mass Spectrometry, 19, 1442-1448.

Yu, Y.-L., Jiang, Y., Chen, M.-L., \& Wang, J.-H. (2011). Lab-on-valve in the miniaturization of analytical systems and sample processing for metal analysis. TrAC Trends in Analytical Chemistry, 30, 1649-1658.

Zotou, A. (2012). An overview of recent advances in HPLC instrumentation. Central European Journal of Chemistry, 10, 554-569. 